\title{
Obstructive sleep apnea-hypopnea syndrome and cognitive impairments in the elderly
}

\author{
Shuling Song ${ }^{1}$, Jin $\operatorname{Tan}^{1}$, Yuyang Miao ${ }^{2}$ and Qiang Zhang ${ }^{1, a}$ \\ ${ }^{1}$ Department of Geriatrics, Tianjin Medical University General Hospital; Tianjin Geriatrics Institute, Tianjin, China \\ ${ }^{2}$ Tianjin Medical University, Tianjin, China
}

\begin{abstract}
Obstructive sleep apnea-hypopnea syndrome (OSAHS) is a common sleep-related breathing disorder that is associated with significant morbidity and mortality. It has received increasing attention that neurocognitive deficits occur with a high frequency in OSAHS. However, it is rarely known that OSAHS impacts on cognition in the elderly in whom an increased prevalence of OSAHS is present. In this review we consider recent studies in the association between OSAHS and cognitive impairments, with specific interest in the older population. Firstly, we elucidate the characteristics of OSAHS and OSAHS-related cognitive impairments in the older patients. Many studies have showed that the prevalence of OSAHS increases with age and it is higher in the elderly than other population. Moreover, OSAHS is associated with higher incidence of comorbidities and increased risk of clinical deterioration in the elderly, especially the neurocognitive impairments which even can develop dementia. Subsequently, we discuss the possible reasons of cognitive impairments that caused or aggravated by OSAHS in the elderly. The intermittent hypoxia (IH)-related disturbances of homeostasis such as oxidative stress, inflammation, and age-related changes such as the changes of sleep architecture, the declined expression level of anti-aging gene, medical comorbidities and polypharmacy, may be both contribute to the increased risk of cognitive impairments in the older patients with OSAHS.
\end{abstract}

\section{Introduction}

OSAHS is a sleep-related breathing disorder characterized by repetitive episodes of airflow cessation resulting in IH and sleep fragmentation which disrupts the sleep architecture and causes an overall poor quality of sleep. Subsequently, daytime functioning is significantly impaired, including excessive sleepiness, fatigue and mood problems [1]. Moreover, OSAHS is always accompanied by multisystem diseases, such as hypertension, arrhythmia, ischemic stroke, diabetes mellitus and so on [2]. Therefore, OSAHS and OSAHS-related multisystem injuries have attracted more and more attention, especially

\footnotetext{
${ }^{a}$ Corresponding author: Zhang Qiang, zhangqiangyulv@163.com

This work was supported by National Natural Science Foundation of China (Grant NO.81370183), Tianjin Natural Science Foundation (Grant No.14JCYBJC27800), National Clinical Key Subject Construction Project of NHFPC Fund and National Training Program of Innovation and Entrepreneurship for Undergraduates (Grant No.201510062005).
} 
the neurocognitive impairments. A wide range of neurocognitive impairments have been identified in OSAHS patients, from attention to memory and executive functions, someone even develop dementia [3].

Recently, a study researched the relevance of sleep disordered breathing (SDB), hypoxia, and risk of mild cognitive impairment (MCI) and dementia in older women [4]. It is a prospective study performed in 298 older women without dementia, founding that $44.8 \%$ of women with SDB developed MCI or dementia after a mean of 4.7 years of follow-up, compared to $31.1 \%$ of women without SDB. After multivariate adjustments, the presence of SDB was still associated with increased risk of developing subsequent MCI or dementia (odd ratio [OR]: 1.85; 95\% confidence interval [CI]: 1.11-3.08). These results suggest that SDB is associated with increased risk of developing cognitive impairments in the older women. It is noteworthy that what on earth the characteristics of OSAHS and OSAHS-related cognitive impairments in the elderly are. In this review, we summarize the current knowledge about characteristics of OSAHS and OSAHS-related neurocognitive impairments in the elderly, and particularly discuss the possible reasons that increase the risk of developing neurocognitive impairments in the older patients with OSAHS.

\section{Characteristics of OSAHS in the elderly}

\subsection{Higher prevalence}

OSAHS, the most common sleep apnea-hypopnea syndrome, can be suffered by population of all ages including children, youth, middle-aged and old people. Although estimates of OSAHS prevalence vary by age, sex, and diagnostic criteria, OSAHS tends to be more common in older adults. A higher prevalence of OSAHS of $38 \%$ up to $63.7 \%$ was reported in the elderly [5-7], compared with the middle-age adults whose prevalence of OSAHS ranged from $2 \%$ to $4 \%$ [8]. The prevalence of OSAHS increases most proportionally with age. In a study performed in 1042 volunteers aged 20 to 80 years old, the prevalence of OSAHS of 20-29y, 30-39y, 40-49y, 50-59y, 60-69y, 70-80y was 7.4\%, $24.2 \%, 37.7 \%, 49.2 \%, 60.2 \%$ and $86.9 \%$, respectively. And the risk of OSAHS also increased with age, reaching $\mathrm{OR}=34.5(95 \% \mathrm{CI}=18.5-64.2, \mathrm{P}<0.001)$ for $60-80$ years old compared with the $20-29$ years old [9].

The higher prevalence of OSAHS in the elderly may be explained by changes in the anatomy or function of the upper airway. The pharyngeal collapsibility is increased with age [10], and the muscular endurance of upper airway is decreased in the elderly [11]. In addition, ventilatory control instability [12] and disordered sleep structure [13], predisposed by sleep fragmentation and resultant frequent shifts from sleep to arousal and vice versa, might contribute to the occurrence of OSAHS in elderly.

\subsection{Longer duration of apnea-hypopnea}

It has been demonstrated that the apnea-hypopnea duration of OSAHS increases with age, and it is longer in the elderly with OSAHS. One study reported that the duration of apnea-hypopnea events was longer in the elderly compared to young and middle-aged OSAHS patients, and the age-related changes were speculated to affect the long duration of apnea-hypopnea events in the older patients, such as increased propensity for pharyngeal collapse, increased deposition of parapharyngeal fat and decreased partial arterial pressure of oxygen [14]. The other study also found that the duration of apnea-hypopnea events was longer in the older patients and it increased significantly with age [15]. The authors explained these findings by breakdown in sensing apnea-hypopnea events and reduction in the stimulus triggering arousals in the older patients with OSAHS. These changes related to age could be associated with the longer duration of apnea-hypopnea in the older patients with OSAHS, but they require large studies. 


\subsection{Severe multisystem injuries}

OSAHS is associated with cardiovascular, cerebrovascular, metabolic, respiratory, digestive and urinary diseases, resulting in multisystem injuries, especially in the elderly whose impairments are more common and more severe.

OSAHS has been related to several cardiovascular comorbidities, such as ischemic heart disease, congestive heart failure, cardiomyopathy and arrhythmias. The older patients with OSAHS have higher risks of occurrence cardiovascular diseases, severe OSAHS even lead to cardiovascular death. One study followed up 372 normotensive older subjects (mean age, 68.2y) 3 years, showed that an $\mathrm{AHI} \geq 30$ per hour was independently related to incident arterial hypertension $(\mathrm{P}=0.02 ; \mathrm{OR}=1.8 ; 95 \%$ $\mathrm{CI}=1.1-2.8$ ), which suggested that severe OSAHS was associated with new-onset arterial hypertension in normotensive elderly [16]. Another study about the relevance between SDB and arrhythmias, showed that complex ventricular ectopy was associated most strongly with OSAHS [17]. In a prospective study performed in 939 elderly $(\geq 65 \mathrm{yr})$, the fully adjusted hazard ratios for cardiovascular mortality were $2.25(\mathrm{CI}=1.41-3.61)$ for the untreated severe OSAHS group, and 1.38 $(\mathrm{CI}=0.73-2.64)$ for the untreated mild to moderate OSAHS group when compared with the control group [18]. These results suggest that severe OSAHS not treated is associated with rised risk of cardiovascular death in the elderly.

In a retrospective cohort study performed in 250,907 patients hospitalized for pneumonia, 15,569 $(6.2 \%)$ of patients were diagnosed with OSAHS, who had a higher prevalence of chronic pulmonary disease when compared with who not with OSAHS (68\% vs 47\%). After adjustments for demographics, comorbidities, admitting physician specialty, hospital type and the effects of clustering, OSAHS was associated with a rised risk of transfer to intensive care $(\mathrm{OR}=1.54 ; 95 \% \mathrm{CI}=1.42-1.68)$ and intubation $(\mathrm{OR}=1.68 ; 95 \% \mathrm{CI}=1.55-1.81)$ on or after the third hospital day [19]. Collectively, these findings provide evidence to support the view that OSAHS increases the susceptibility to respiratory disease, and hypoxia becomes more severe when OSAHS coexists with these diseases, which increases the risk of clinical deterioration.

Evidences from population, clinic-based, and laboratory studies had indicated that OSAHS can increase the risk of developing of insulin resistance, glucose intolerance and type 2 diabetes [20]. Moreover, OSAHS can contribute independently to the development of nocturia, and the frequency of nocturia increases as the severity of OSAHS increase [21]. It is reported that OSAHS patients also suffer from nocturnal gastroesophageal reflux and laryngopharyngeal reflux, which can lead to injury of esophageal mucosal and ulcer [22]. In conclusion, multisystem diseases associated with OSAHS can aggravate the damage that is related to ageing.

\section{Characteristics of cognitive impairments in the elderly with OSAHS}

\subsection{High risk of occurrence}

$\mathrm{IH}$ and sleep fragmentation, the major physiopathologic characteristic of OSAHS, can cause ischemia and hypoxia of brain resulting in increased risk of cerebrovascular accidents, such as ischemic stroke [23], brain infarction [24], mild cognitive impairment and dementia [4].

Multiple studies have been demonstrated that SDB is significantly associated with cognitive impairments. In a study performed in 1389 community-dwelling individuals 60 to 70 years old [25], $10.8 \%$ of subjects of this older sample reported apnea during sleep, and $49.5 \%$ reported snoring. After adjustments for age, gender, educational level, tobacco status, alcohol consumption, depressive symptomatology and number of medications, logistic regression models showed that both snoring and apnea were associated with low scores in the Digit Symbol Substitution Test $(\mathrm{OR}=1.80,95 \%$ $\mathrm{CI}=1.09-2.99$; $\mathrm{OR}=1.58,95 \% \mathrm{CI}=0.87-2.89$, respectively), and in the Trail Making Test $(\mathrm{OR}=2.14$, $95 \% \mathrm{CI}=1.24-3.69 ; \mathrm{OR}=1.88,95 \% \mathrm{CI}=1.04-3.39$, respectively). This study indicated that $\mathrm{SDB}$ was a risk factor for poor cognitive performance in tests requiring visual attention skills. Yaffe et al. [4] also found that the adjusted OR for developing cognitive impairments was 1.85 (95\% CI=1.11-3.08) in 
older women with SDB. All these findings suggest that SDB is associated with a rised risk of developing cognitive impairments in elderly.

\subsection{Severe cognitive impairments}

OSAHS-related cognitive impairments in the elderly are more severe, which are reflected by poor performance in cognitive tests and changes of brain structure. A study comparing the cognitive performance in attention-alertness tests among subjects of different ages, showed that the older patients had poor performance compared with their age-matched controls, while young patients' cognitive performance was similar to their age-matched controls [26]. These findings suggest that cognitive dysfunctions of OSAHS patients are age dependent. Ayalon et al. [27] also showed that older patients had poor performance for immediate word recall and slower reaction time during sustained attention in comparison with older controls, young patients and young controls, and detected a decreased activation of the frontal gyrus, hippocampus and parietal lobe during the tasks by functional magnetic resonance imaging. These results are supported by studies that used structural and functional neuroimaging to examine the brain regions affected in patients with OSAHS with cognitive impairments.

\subsection{More underlying diseases}

It is common that the elderly have more underlying diseases, such as chronic obstructive pulmonary disease (COPD), coronary heart disease and heart failure, which can worsen ischemia/hypoxia of OSAHS patients, and increase the risk of cognitive deficits in the older patients. For example, the occurrence of both COPD and OSAHS in an individual patient has been described as "overlap syndrome", which has a high risk for comorbidities, such as arterial hypertension and diabetes [28].

\section{Possible reasons of cognitive impairments that caused or aggravated by OSAHS in the elderly}

\subsection{Oxidative stress}

$\mathrm{IH}$ is now being recognized as a main factor of contributing to occurrence of OSAHS-related comorbidities. IH promotes oxidative stress by increasing production of reactive oxygen species (ROS) which is associated with cell damage and apoptosis. It has led to the hypothesis that oxidative stress induced by IH is associated with neurocognitive impairments in patients with OSAHS. Xu et al. [29] developed chronic intermittent hypoxia $(\mathrm{CIH})$ mouse model to study the mechanisms of $\mathrm{CIH}$ mediated brain cortical neuronal cell damage. They detected significant increase of ROS level and oxidative stress response markers (c-Fos, c-Jun and NF- $\mathrm{kB}$ ) in brain cortex of mice exposured to CIH. Moreover, $\mathrm{CIH}$ induced caspase-3 activation in cortical neuronal cell. However, transgenic mice, overexpressing $\mathrm{Cu}, \mathrm{Zn}$-superoxide dismutase exposed to $\mathrm{CIH}$, had a low level of ROS and reduced neuronal apoptosis in brain cortex. These results demonstrated that increased level of ROS was associated with neuronal cell damage. The other study also demonstrated that antioxidants intervention can prevent $\mathrm{CIH}$-induced decline in spatial learning during the Morris water maze as well as $\mathrm{CIH}$-induced decrease in superoxide dismutase levels [30]. Taken together, all of these findings indicate that $\mathrm{CIH}$-mediated increased ROS production and subsequently oxidative stress propagation contribute, at least partially, to neuronal apoptosis and neurocognitive impairments.

\subsection{Inflammation}

Recently, lots of studies have showed that OSAHS patients have increased plasma markers of systemic inflammation, and IH is also associated with increased levels of inflammatory cytokines, 
adhesion molecules, and activation of circulating neutrophils in IH rodent model. One study explored the association between OSAHS and inflammation through measured the activity of NF- $\mathrm{BB}$ by electrophoreticmobility shift assay, and the concentration of soluble E (sE)-selectin and soluble vascular cell adhesion molecule-1 (Svcam-1) by enzyme-linked immunosorbent assay in OSAHS patients and control subjects [31]. They found that the activity of NF- $\mathrm{BB}$ and the concentration of sEselectin and Svcam-1 were higher in OSAHS patients than control subjects. Another study also identified a strong association between OSAHS and increased levels of TNF- $\alpha$ and IL-8 [32]. And these inflammatory markers were reduced after continuous positive airway pressure (CPAP) therapy. What's more, it was demonstrated that IH caused low-grade neuroinflammation in the hippocampus of mice, including early but transient elevation of cytokine (IL-1 $\beta$ and RANTES/CCL5), and delayed but long-term changes of microglia which are major inflammatory cells in the central nervous system [33]. Peripheral cytokines, which can penetrate the blood-brain barrier directly via active transport mechanisms or indirectly via vagal nerve stimulation, combine with central cytokines cause endothelial dysfunction and aggravate brain damage [34]. These results suggest that IH-mediated inflammation is associated with neurocognitive deficits.

\subsection{Changes of sleep architecture}

Sleep architecture underwent significant alterations throughout the aging process in the general population. In other words, sleep architecture, especially the sleep-wake cycle, changes significantly in the elderly, which include the decrease of total sleep time, sleep efficiency, percentage of rapid eye movement (REM) sleep and slow wave sleep, and the increase of WASO (night-time spent awake after sleep onset), arousal index, sleep latency, REM sleep latency and the percentage of stages 1 and 2 [35]. It is noteworthy that OSAHS causes REM sleep fragmentation, while REM sleep may play a major role in selective emotional memory processing and recalibrating the brain's responses to emotional events [36]. REM sleep disturbance caused by OSAHS may affect the cognitive functions through the emotional disturbance. Moreover, slow wave activity (SWA) during the non-REM has been postulated to play a role in the abstraction of rules and the integration of knowledge into existing schemas [37], while OSAHS decreases or fragments causes slow wave sleep. Thus, OSAHS can cause the cognitive impairments through such effects on SWA during the non-REM stages. From the above results, we can speculate that sleep stage dysfunction and fragmentation may play an important role in the development of cognitive impairments in the older patients with OSAHS.

\subsection{Anti-aging gene}

Some of diseases are more common in the elderly, such as coronary heart disease, heart failure, stroke and so on, whose common pathophysiological characteristic is ischemia/hypoxia. This phenomenon indicates that the older people have unique vulnerability to ischemia/hypoxia. Moreover, the increased susceptibility to IH in aging rats was also demonstrated through developing IH model [38]. Anti-aging genes, such as sirtuin 1 (Sirt1), Klotho and so on, decrease with age, and they are declined in ischemic/hypoxic diseases. Collectively, all theses findings suggest that vulnerability of the older people to ischemia/hypoxia may be associated with declined expression of anti-aging gene.

Sirt1, the mammalian NAD+-dependent histone deacetylase, is involved in the regulation of different cellular processes, ranging from cell cycle, metabolism, and apoptosis to the cellular energy and redox status. It has been demonstrated that the expression level of Sirt1 decreases with age [39], and low level of Sirt1 is associated with increased susceptibility to ischemic/hypoxic diseases [40]. Shinji et al. detected the expression of Sirt1 mRNA and serum cystatin C, and prevalence of mutations in mitochondrial DNA in young mice and aged mice, respectively [41]. The result showed that the expression of Sirt1 was reduced in aged mice, which was linked to age-associated kidney dysfunction and mitochondrial oxidative damage. However, after long-term calorie restriction, the expression level of Sirt1 was increased, and kidney dysfunction and mitochondrial oxidative damage 
were attenuated. Collectively, these findings indicate that the susceptibility of the elderly to hypoxia is associated with decreased level of Sirt1.

As a recently discovered anti-aging gene, Klotho was originally identified in a mutant mouse that exhibited multiple disorders resembling human premature-aging syndrome, including shortened life span, arteriosclerosis, skin atrophy, osteoporosis and emphysema [42]. Yamamoto et al. detected the urinary 8-hydroxydeoxyguanosine (8-OHdG, a biological marker of in vivo oxidative DNA damage) of Klotho-overexpressing transgenic mice and wild-type mice, and found that the average urinary 8$\mathrm{OHdG}$ excretion in transgenic mice was one-half of that in wild-type mice [43]. Furthermore, the authors found that the transgenic mice survived a challenge with a lethal dose of paraquat significantly longer than wild-type mice. All these results indicate that Klotho can increase resistance to oxidative stress.

The declined expression level of anti-aging gene is not only associated with increased susceptibility to ischemic/hypoxic diseases, but also related to cognitive impairments. Michán et al. detected that Sirt1 was expressed in hippocampal neurons by using a mouse specific anti-Sirt1 antibody [44]. Then they found that Sirt1 knockout mice exhibited poor cognitive performance, including immediate memory, classical conditioning and spatial learning. These findings suggest that Sirt1 plays an important role in maintaining normal learning, memory and synaptic plasticity. Recently, it also has been demonstrated that Klotho can increase the expression of erythropoietin receptor (EpoR) in neuronal cell and enhance Epo-mediated neuroprotection [45].

Based on above findings, we speculate that the more frequent and more severe OSAHS-related cognitive deficits in the elderly may be associated with the declined expression level of anti-aging gene.

\subsection{Others}

Some diseases that are common in the elderly, such as obesity, hypertension, diabetes, are both connected with increased risk of OSAHS and cognitive impairments. The relationship between obesity and OSAHS is bidirectional, that is, obesity and OSAHS reinforce each other [46]. In a study aimed to evaluate the impact of obesity on cognition of patients with OSAHS, the Total intelligence quotient and the Performance intelligence quotient scores were significantly lower in obese OSAHS patients with poor performance on cognitive tests, compared to OSAHS patients and normal controls [47]. These results suggest that obesity has a synergic and additive action with that exerted by OSAHS, aggravating the cognitive impairments. The effects of hypertension on cognition and OSAHS are similar to the impacts of obesity, which interact with OSAHS and both are related to cognitive impairments. It also has been found that both OSAHS and hypertension are associated with worse auditory verbal memory and executive function in the elderly [48]. The coexistence of many diseases can increase risk of underlying OSAHS and aggravate cognitive impairments, which provide further support to the hypothesis that medical comorbidities may play an important role in the occurrence of cognitive impairments in older patients with OSAHS.

Since the multisystem diseases are common in the elderly, so polypharmacy has become common in the elderly who are more likely to develop cognitive impairments associated with medications. Psychoactive drugs, antidepressants and anticonvulsants can lead to dementia and delirium. Moreover, non-psychoactive drugs, such as antibiotics, cardiac medications and histamine $\mathrm{H} 2$ receptor antagonists may lead to acute or chronic cognitive impairments [49]. It is noteworthy that certain medications can worsen underlying OSAHS. For example, the patients who taken atypical antipsychotics (AAs) had significantly elevated OR compared with the patients who didn't take AAs (4.5 vs 1.9) [50]. The impact of OSAHS on cognition is greater in the elderly than in other OSAHS populations may be associated with polypharmacy, but it requires large studies to delineate. 


\section{Conclusion}

The presence of OSAHS is higher in the elderly. And the damage of nervous system, especially cognitive impairments, is more frequent and more severe in the older patients. However, the effectiveness of treatments of OSAHS and OSAHS-related cognitive impairments is little known in the older patients. So the study of pathogenesis of OSAHS and OSAHS-related cognitive impairments is of great significance for clinical treatment in the older patients. IH-related disturbance of homeostasis and age-related changes may provide more space for the exploration of new treatments in the elderly who have OSAHS. But these mechanisms described above of cognitive impairments induced by OSAHS in the elderly are unclear. Further studies are needed to determine the correlation between OSAHS and cognitive impairments, which might accelerate the development of therapeutic interventions in OSAHS and OSAHS-related cognitive impairments in the elderly.

\section{References}

1. T.J. Vaessen, S. Overeem, M.M. Sitskoorn, Sleep. Med. Rev 19, 51 (2015).

2. N.A Dewan, F.J. Nieto, V.K. Somers, Chest 147, 266 (2015).

3. R.S. Bucks, M. Olaithe, P. Eastwood, Respirology 18, 61 (2013).

4. K. Yaffe, A.M. Laffan, S.L. Harrison, S. Redline, A.P. Spira, K.E. Ensrud, S. Ancoli-Israel, K.L. Stone, JAMA 306, 613 (2011).

5. P. Johansson, U. Alehagen, E. Svanborg, U. Dahlström, A. Broström, Age. Ageing 41,468 (2012)

6. E. Sforza, F. Chouchou, P. Collet, V. Pichot, J.C. Barthélémy, F. Roche, Eur. Respir. J 37, 1137 (2011).

7. S.S. Ng, T.O. Chan, K.W. To, K.K Chan, J. Ngai, A. Tung, F. W. Ko, D.S. Hui, PLoS One 10 (2015).

8. T. Young, M. Palta, J. Dempsey, J. Skatrud, S. Weber, S. Badr, N. Engl. J. Med 328, 1230 (1993)

9. S. Tufik, R. Santos-Silva, J. A. Taddei, L. R. Bittencourt, Sleep. Med 11, 441 (2010) .

10. M. Eikermann, A. S. Jordan, N. L. Chamberlin, S. Gautam, A. Wellman, Y. L. Lo, D. P. White, A. Malhotra, Chest 131, 1702 (2007).

11. C. Worsnop, A. Kay, Y. Kim, J. Trinder, R. Pierce, J. Appl. Physiol 88, 1831 (2000).

12. S. Celle, R. Peyron, I. Faillenot, V. Pichot V, M. Alabdullah, J. M. Gaspoz, B. Laurent, J.C. Barthélémy, F. Roche F, Hum. Brain. Mapp 30, 2090 (2009).

13. W. Moraes, R. Piovezan, D. Poyares, L. R. Bittencourt, R. Santos-Silva, S. Tufik, Sleep. Med 15, 401 (2014).

14. E. George, V. Katerina, S. Maria, B. Lambros, N. Konstantina, G. Dimitrios, Sleep. Disord, 2012 (2012) .

15. J. C. Ware, R. H. McBrayer, J. A. Scott, Sleep 23, 165 (2000) .

16. M. Guillot, E. Sforza, E. Achour-Crawford, D. Maudoux, M. Saint-Martin, J. C. Barthélémy, F. Roche, Sleep. Med 14, 838 (2013).

17. R. Mehra, K. L. Stone, P. D. Varosy, A. R. Hoffman, G. M. Marcus, T. Blackwell, O. A. Ibrahim R. Salem, S. Redline, Arch. Intern. Med 169, 1147 (2009).

18. M. A. Martínez-García, F. Campos-Rodríguez, P. Catalán-Serra, J. J Soler-Cataluña, C. AlmeidaGonzalez, I. De la Cruz Morón, J. Durán-Cantolla J, J. M. Montserrat, Am. J. Respir. Crit. Care. Med 186, 909 (2012).

19. P. K. Lindenauer, M. S. Stefan, K. G. Johnson, A. Priya, P. S. Pekow, M. B. Rothberg, Chest 145, 1032 (2014).

20. R. N. Aurora, N. M. Punjabi, Lancet. Respir. Med 1, 329 (2013).

21. S. Ayik, K. Bal, G. Akhan, Turk. J. Med. Sci 44, 249 (2014).

22. S. K. Wise, J. C. Wise, J. M. DelGaudio, Otolaryngol. Head. Neck. Surg, 135, 253 (2006).

23. S. Redline, G. Yenokyan, D. J. Gottlieb, E. Shahar, G. T. O'Connor, H. E. Resnick, M. DienerWest, M. H. Sanders, P. A. Wolf, E. M. Geraghty, T. Ali, M. Lebowitz, N. M. Punjabi, Am. J. Respir. Crit. Care. Med 182, 269 (2010). 
24. K. Minoguchi, T. Yokoe, T. Tazaki, H. Minoguchi, N. Oda, A. Tanaka, M. Yamamoto, S. Ohta, C. P. O'Donnell, M. Adachi, Am. J. Respir. Crit. Care. Med 175, 612 (2007).

25. M. J. Dealberto, N. Pajot, D. Courbon, A. Alpérovitch, J. Am. Geriatr. Soc 44, 1287 (1996).

26. M. Alchanatis, N. Zias, N. Deligiorgis, I. Liappas, A. Chroneou, C. Soldatos, C. Roussos, Sleep. Breath 12, 17 (2008).

27. L. Ayalon, S. Ancoli-Israel, S. P. Drummond, Am. J. Respir. Crit. Care. Med 182, 413 (2010)

28. E. H. Steveling, C. F. Clarenbach, D. Miedinger, C. Enz, S. Dürr, S. Maier, N. Sievi, S. Zogg, J. D. Leuppi, M. Kohler, Respiration 88, 451 (2014).

29. W. Xu, L. Chi, B. W. Row, R. Xu, Y. Ke, B. Xu, C. Luo, L. Kheirandish, D. Gozal, R. Liu, Neuroscience 126, 313 (2004).

30. L. Hui-guo, L. Kui, Z. Yan-ning, X. Yong-jian, Sleep. Med 11, 205 (2010).

31. A. K. Htoo, H. Greenberg, S. Tongia, G. Chen, T. Henderson, D. Wilson, S. F. Liu, Sleep Breath 10, 43 (2006).

32. S. Ryan, C. T. Taylor, W. T. McNicholas, Am. J. Respir. Crit. Care. Med 174, 824 (2006).

33. E. Sapin, C. Peyron, F. Roche, N. Gay, C. Carcenac, M. Savasta, P. Levy, M. Dematteis, Sleep 38 , 1537 (2015).

34. C. J. Wilson, C. E. Finch, H. J. Cohen, J. Am. Geriatr. Soc 50, 2041 (2002).

35. W. Moraes, R. Piovezan, D. Poyares, L. R. Bittencourt, R. Santos-Silva, S. Tufik, Sleep. Med 15, $401(2014)$.

36. A. N. Goldstein, M. P. Walker, Annu. Rev. Clin. Psychol 10, 679 (2014).

37. N. Landmann, M. Kuhn, H. Piosczyk, B. Feige, C. Baglioni, K. Spiegelhalder, L. Frase, D. Riemann, A. Sterr, C. Nissen, Sleep Med. Rev 18, 531 (2014).

38. Gozal D, Row BW, Kheirandish L, R. Liu, S. Z. Guo, F. Qiang, K. R. Brittian, J. Neurochem 86, 1545 (2003).

39. H. Gong, J. Pang, Y. Han, Y. Dai, D. Dai, J. Cai, T. M. Zhang, Mol. Med. Rep 10, 3296 (2014)

40. C. Tong, A. Morrison, S. Mattison, S. Qian, M. Bryniarski, B. Rankin, J. Wang, D. P. Thomas, J. Li, FASEB. J 27, 4332 (2013).

41. S. Kume, T. Uzu, K. Horiike, M. Chin-Kanasaki, K. Isshiki, S. Araki, T. Sugimoto, M. Haneda, A. Kashiwagi, D. Koya, J. Clin. Invest 120, 1043 (2010) .

42. M. Kuro-o, Y. Matsumura, H. Aizawa, H. Kawaguchi, T. Suga, T. Utsugi, Y. Ohyama, M. Kurabayashi, T. Kaname, E. Kume, H. Iwasaki, A. Iida, T. Shiraki-Iida, S. Nishikawa, R. Nagai, Y. I. Nabeshima, Nature 390, 45 (1997).

43. M. Yamamoto, J. D. Clark, J. V. Pastor, P. Gurnani, A. Nandi, H. Kurosu, M. Miyoshi, Y. Ogawa, D. H. Castrillon, K. P. Rosenblatt, M. Kuro-o, J. Biol. Chem 280, 38029 (2005).

44. S. Michán, Y. Li, M. M. Chou, E. Parrella, H. Ge, J. M. Long, J. S. Allard, K. Lewis, M. Miller, W. Xu, R. F. Mervis, J. Chen, K. I. Guerin, L. E. Smith, M. W. McBurney, D. A. Sinclair, M. Baudry, R. de Cabo, V. D. Longo, J. Neurosci 30, 9695 (2010).

45. M. F. Cheng, L. J. Chen, H. S. Niu, T. T. Yang, K. C. Lin, J. T. Cheng, Acta. Neurobiol. Exp (Wars) 75, 60 (2015).

46. S. Ryan, S. J. Crinion, W. T. McNicholas, QJM 107, 949 (2014).

47. O. Vitelli, A. Tabarrini, S. Miano, J. Rabasco, N. Pietropaoli, M. Forlani, P. Parisi, M. P. Villa, Sleep Med 16, 625 (2015) .

48. L. M. Kinoshita, J. A. Yesavage, A. Noda, B. Jo, B. Hernandez, J. Taylor, J. M. Zeitzer, L. Friedman, J. K. Fairchild, J. Cheng, W. Kuschner, R. O'Hara, J. E. Holty, B. K. Scanlon, Sleep Breath 16, 1201 (2012).

49. M. Shinohara, M. Yamada, Brain Nerve 64, 1405 (2012).

50. A. Shirani, S. Paradiso, M. E. Dyken, Sleep Med 12, 591 (2011). 